\section{Challenging Times for Children With Transfusion-dependent Thalassemia Amid the COVID-19 Pandemic}

A novel coronavirus disease (COVID-19), caused by severe acute respiratory syndrome coronavirus 2 (SARSCoV-2), originally from Wuhan, China, has now become a pandemic. To curtail its spread, many countries, including India, have taken a judicious decision to have nationwide lockdowns restricting movements of citizens. However, this has made it difficult for children with many chronic conditions to continue their therapy.

Children with thalassemia major require periodic blood transfusions. In India, almost half of children with $\beta$-thalassemia major under-transfused [1]. With the lockdown in our country, patients and their parents would find it difficult to visit their routine clinics for blood transfusions. Moreover, the lockdown has drastically reduced the number of voluntary blood donations, thereby creating a shortage at blood banks. Despite the cancellation of all elective surgeries, blood units available for transfusion are less [2]. Although viral RNA has been detected in the plasma/serum of COVID-19 patients, the present data do not suggest the risk of transfusion transmission of SARS-CoV-2. However, certain International organizations have advised deferral of blood donation for 21 days after possible exposure to a confirmed case and for at least 28 days after symptom resolution in a positive case [3]. In addition, patients on iron chelation therapy may find it difficult to procure the drugs amid lockdown.

Eventually, there is an underlying risk of these children contracting COVID-19. Unlike sickle cell anemia, children with thalassemia are usually not at an increased risk of fatal pulmonary complications due to COVID-19. However, splenectomy and underlying comorbidities secondary to iron overload, notably secondary diabetes mellitus, cardiomyopathy and chronic liver disease, may increase the risk of complications and mortality in COVID-19 [4].
Certain solutions do exist. Blood transfusions could be carried at any nearest convenient healthcare facility instead of routine transfusion clinics. Healthcare authorities should strengthen mobile unit services for facilitating blood donation at doorstep while ensuring stringent precautions. Till blood stocks replenish, caregivers can bring a voluntary healthy donor at the time of transfusion. Physicians should educate children and caregivers about need for strict social distancing, hand hygiene and common symptoms of COVID-19. Teleconsultations may play a role in this regard. Children with associated comorbidities must be more cautious. Good glycemic control in patients with secondary diabetes should be ensured. Underlying subclinical hypoadrenalism should be considered in every thalassemic child with suspected COVID-19 and supplemented with stress-dose of glucocorticoids.

Funding: None; Competing interests: None stated. Published online: April 07, 2020; PII: S097475591600155

URMILA YADAV ${ }^{1}$ AND RIMESH PAL ${ }^{2 *}$

${ }^{1}$ National Institute of Nursing Education and ${ }^{2}$ Department of Endocrinology,

Post Graduate Institute of Medical Education and Research, PGIMER, Chandigarh, India. rimesh.ben@gmail.com

\section{REFERENCES}

1. Shah N, Mishra A, Chauhan D, Vora C, Shah N. Study on effectiveness of transfusion program in thalassemia major patients receiving multiple blood transfusions at a transfusion centre in Western India. Asian J Transfus Sci. 2010;4:94-8

2. Covid-19: Lockdown creates acute shortage at blood banks. Available from: https:// timesofindia.indiatimes.com/india/ covid-19-lockdown-creates-acute-shortage-at-bloodbanks/articleshow/74958205.cms. Accessed April 3, 2020.

3. Chang L, Yan Y, Wang L. Coronavirus Disease 2019: Coronaviruses and Blood Safety. Transfus Med Rev [Internet]. 2020 [cited 2020 Apr 1]; Available from: https:/ /linkinghub.elsevier.com/retrieve/pii/S0887796320300146. Accessed April 3, 2020.

4. Thalassaemia International Federation. The covid-19 pandemic and haemoglobin disorders. Available from: https://thalassaemia.org.cy/wp-content/uploads/2020/03/ COVID-19-pandemic-and-haemoglobin-disorders_V2. pdf. Accessed April 3, 2020. 